\title{
NOTE ON ORTHOGRAPHY
}

MODERN TURKISH IS WRITTEN with the Latin alphabet. Most letters loosely correspond to characters found in English, but there are a few differences. The Turkish $c$ is pronounced like the English $j$ as in jam. $J$ is pronounced like the final "ge" of the French rouge. Ç is pronounced "ch" as in child. Ş is "sh" as in show. $\dot{I}$ and $\imath$ (i.e., with and without a dot) are comparable to "ee" and "uh" respectively. $\breve{G}$ lengthens the vowel preceding it but is not pronounced separately. Umlauts (as in $\ddot{o}$ and $\ddot{u}$ ) are akin to those in German.

Throughout this book, I make use of modern standard Turkish orthography for most technical Islamic terms. For instance, I use cemaat rather than jama $\bar{a} a$. Where relevant, I note the standard transliteration of Arabic in parenthesis. Exceptions to the use of Turkish orthography include words that are commonly represented in English: I use Shi 'i rather than either Shī î (formally transliterated Arabic) or Şii (Turkish). I have also rendered proper names of figures from early Islamic history with conventional English spellings, for example, Husayn rather than Hüseyin (Turkish). All translations are my own unless noted. 

The Reckoning of Pluralism 
